\title{
Bases y desafíos de la aplicación de convenios de desempeño en la educación superior de Chile
}

\author{
Basis and challenges of the application of performance \\ agreements in Chilean higher education
}

\author{
Ricardo Reich Albertz ${ }^{1} \quad$ Flora Machuca ${ }^{2} \quad$ Daniel López Stefoni $^{3} \quad$ Juan P. Prieto $^{4}$ \\ Juan Music $^{5} \quad$ Emilio Rodríguez-Ponce ${ }^{6} \quad$ Jorge Yutronic $^{7}$ \\ Recibido 15 de noviembre de 2010, aceptado 1 de abril de 2011 \\ Received: November 15, $2010 \quad$ Accepted: April 1, 2011
}

\begin{abstract}
RESUMEN
El presente artículo analiza el modelo de financiamiento utilizado por el Ministerio de Educación de Chile. Este modelo considera mecanismos que incentivan la implementación o mejoramiento del desempeño estratégico de las instituciones, así como el eficaz empleo de unidades de análisis institucional, tanto para efectos del desarrollo de programas y proyectos, como para la gestión institucional y la alimentación del Sistema de Información de la Educación Superior (SIES).

En Chile, a través de la experiencia lograda con el Programa de Mejoramiento de la Calidad y el Desempeño de la Educación Superior (MECESUP) para promover los objetivos de política pública, el Ministerio de Educación ha dado lugar al desarrollo experimental de un nuevo instrumento de asignación de recursos basado en resultados y en sus impactos académicos y estratégicos, este instrumento se denomina: Convenios de Desempeño. Los resultados de la experiencia piloto indican que son un instrumento de financiamiento eficaz y, principalmente, son un incentivo y un medio para lograr mejoramientos relevantes en las instituciones y el sistema de educación superior. La experiencia ganada con la ejecución de estos convenios permite sugerir, en este trabajo, aspectos que deben ser perfeccionados para lograr dar sustentabilidad a este instrumento como política pública y lograr una visión y estrategia integradora de mejoramiento de la calidad, en el quehacer integral de las universidades.
\end{abstract}

Palabras clave: Modelo de financiamiento, convenios de desempeño, sistema de información corporativo, sistema chileno de educación superior, planes de mejoramiento.

\section{ABSTRACT}

This paper analyzes the funding model used by the Chilean Ministry of Education. The model considers mechanisms to encourage the implementation or enhancement of institutional performance and the use of analysis units, both for the development of programs and projects, and the institutional management and the feeding of the Higher Educational Information System (SIES).

In Chile, through the experience obtained with the Higher Education Quality and Performance Enhancement Program (MECESUP) to promote the objectives of the public policy, the Ministry of Education has created the experimental development of a new instrument for assigning resources based on results, Performance Agreements. Results indicate that they are an efficient funding instrument and, mainly, are an incentive

\footnotetext{
Director Ejecutivo del MECESUP. Ministerio de Educación de Chile. Santiago, Chile.

Ministerio de Educación de Chile. Santiago, Chile.

Vicerrector de Investigación y Desarrollo de la Universidad de Playa Ancha. Valparaíso, Chile.

Vicerrector Académico de la Universidad de Viña del Mar. Investigador del Programa Anillo de Investigación en Ciencias Sociales de CONICYT (SOC-01). Viña del Mar, Chile.

Profesor Titular de la Universidad Católica del Norte. Antofagasta, Chile.

Profesor Titular de la Universidad de Tarapacá. Arica, Chile. Investigador Responsable del Proyecto FONDECYT 1090116.

E-mail: erodrigu@uta.cl

7 Consultor. Ministerio de Educación de Chile. Santiago, Chile.
} 
and a means to get important enhancements in higher education institutions and the higher education system. Experience gained with the application of these agreements, suggests, in this paper, aspects to perfect in order to provide sustainability to this instrument as a public policy, and get an integrating vision and strategy of quality enhancement in the overall work of universities.

Keywords: Funding model, performance agreements, corporate information system, Chilean higher educational system, improvement plans.

\section{INTRODUCCIÓN}

El sistema chileno de educación superior tiene características muy particulares que lo diferencian de la mayoría de los países de la OECD (Organization for Economic Co-operation and Development). Una de las principales es el grado de autonomía de las instituciones, que se expresa en su amplia libertad para definir aspectos claves como la oferta de vacantes, programas académicos, aranceles, plantas académicas, salarios de los profesores, entre otros. A esta autonomía se suma una gran diversidad institucional, un esquema mixto de provisión, la coordinación del sistema principalmente por el mercado y un modelo de financiamiento que ha estado avanzando desde un financiamiento bimodal, basal histórico y de la demanda, a uno multimodal con una mayor variedad de instrumentos y con creciente énfasis en los resultados, su divulgación con transparencia y la rendición de cuentas públicas. El modelo tiene un significativo componente de financiamiento privado a través de aranceles (rol de las familias); en Chile, es proporcionalmente uno de los más altos en el mundo, junto a Corea.

Uno de los más serios problemas que opaca la transparencia del sistema de educación superior chileno es la falta de información confiable, relevante y oportuna, tanto del sistema como de las instituciones individualmente. El levantar información de calidad para el sistema de educación superior, para las propias instituciones y, en general, para los diversos actores con intereses en él, ha sido lento y dificultoso.

La reacción del Ministerio de Educación ante el escenario actual de los sistemas de información corporativo y de la escasa gestión en base a evidencia ha sido incentivar la instalación o mejoramiento de unidades de análisis institucional, tanto para efectos del desarrollo de programas y proyectos como para la gestión institucional y la alimentación del
Sistema de Información de la Educación Superior (SIES), residente en el Ministerio de Educación. No obstante, sobre este punto, los informes de evaluación advierten todavía falta de capacidad para sistematizar y usar la información generada al interior de las instituciones de educación superior [1-2].

La experiencia internacional ha desarrollado diversos instrumentos de financiamiento público que buscan básicamente tres grandes objetivos de política para los sistemas: i) aumentar el acceso y la equidad, ii) aumentar la eficiencia externa y iii) aumentar la eficiencia interna del sistema.

Particularmente es a través del financiamiento público a la oferta que se busca incorporar mecanismos de mayor racionalidad económica, asociando los fondos con resultados. En Chile, éste se canaliza básicamente a través de tres instrumentos: Aporte Fiscal Directo (AFD), Aporte Fiscal Indirecto (AFI) y Programa de Mejoramiento de la Calidad y el Desempeño de la Educación Superior (MECESUP), siendo este último el único de carácter concursable y que compromete resultados. La experiencia del Programa MECESUP en promover estos tres objetivos de política ha dado lugar, el año 2007, al desarrollo experimental de un nuevo instrumento de asignación de recursos basado en resultados: los Convenios de Desempeño. Su propósito general es aumentar la efectividad del gasto público, alinear los objetivos institucionales con los objetivos de interés nacional, favorecer la rendición de cuentas pública, así como asociar el mejoramiento del desempeño institucional con el financiamiento.

En sus grandes orientaciones, este nuevo instrumento coincide con una de las principales sugerencias del Consejo Asesor Presidencial de Educación Superior: "es mejor un sistema de asignación de recursos centrados en resultados y mediante mecanismos competitivos" (Consejo Asesor, 2008). En la práctica, responde a la necesidad de diseñar e implementar 
otros instrumentos de financiamiento diferentes a los existentes, ya que ciertos problemas estructurales del sistema de educación superior aún subsisten y no se resuelven (como los desempeños docentes y de aprendizaje en el nivel de pregrado).

A nivel internacional existen, en general, tres modalidades de asignación de recursos públicos a la oferta: el financiamiento inercial, el uso de fórmulas y los contratos [3]. La primera modalidad corresponde a la más tradicional de asignación de recursos basales estables, lo que en Chile correspondería al AFD. Las otras dos modalidades asignan en general un presupuesto secundario.

El financiamiento mediante fórmulas presenta diversas modalidades, algunas basadas en los insumos, como el número de estudiantes o el número de académicos con doctorado. Otras fórmulas consideran el costo por estudiante o bien objetivos nacionales, una suerte de subsidio preferente, como carreras específicas de alta prioridad nacional o regional, o bien asociadas al tipo de estudiante que matriculan, típicamente provenientes de sectores subrepresentados. También existen fórmulas que tienen componentes asociados a resultados, como el número de titulados, o la cantidad de créditos impartidos en docencia o el número de publicaciones ISI (en el caso chileno, las fórmulas son básicamente el AFI y el 5\% recurrente del AFD).

El financiamiento basado en resultados premia a las instituciones por su desempeño real en lugar del prometido, utilizando indicadores que reflejan los intereses de la política pública. Existen dos mecanismos de asignación de fondos por resultados de particular relevancia. Los fondos competitivos que financian proyectos de mejoramiento académico y que son evaluados por pares, como es el caso del Fondo de Innovación Académica (FIAC) y los Convenios de Desempeño piloto del Programa MECESUP (CDs), en los cuales se concuerdan objetivos medibles entre las instituciones y el gobierno.

Los contratos de desempeño implementados en diversos países y varios estados de Estados Unidos ${ }^{8}$ son de disímil características, algunos se basan en estándares e indicadores, otros establecen objetivos

8 Alemania, Austria, Canadá, Cataluña, Estados Unidos (en un número de estados), Finlandia y Francia. generales para el sistema y objetivos específicos para cada institución, algunos representan un porcentaje pequeño del aporte fiscal y en otros casos es la totalidad. En concreto, la mayoría de estos señalan con claridad los ámbitos en que son útiles, como el mejoramiento de indicadores y eficiencia docentes (tasas de retención y graduación oportuna), el potenciamiento de la productividad científico-tecnológica (número de publicaciones, impacto y valorización para la innovación) y el mejoramiento de la gestión institucional (indicadores financieros y de infraestructura, entre otros). Los CDs son un instrumento de financiamiento, pero principalmente son un incentivo y medio para lograr mejoramientos relevantes en las instituciones y el sistema de educación superior. Desde el punto de vista práctico, constituyen una forma de trabajo distinto entre las agencias de financiamiento (como el Ministerio de Educación), ya que negocian y se ponen de acuerdo en torno a objetivos comunes.

Existen algunos factores de diseño que es necesario tener en cuenta. ¿Qué proporción de los recursos asignar por esta vía? ¿Qué indicadores se deben usar para medir el desempeño?Y ¿cómo tratar a las instituciones que presentan un mal desempeño? [5-6]. Otras consideraciones de importancia, a la hora de introducir mecanismos de asignación de recursos basados en resultados, se refieren a la existencia de información confiable y oportuna a nivel de sistema y de las propias instituciones; sin esto se torna difícil evaluar el desempeño institucional. También es relevante la existencia de adecuadas prácticas de gestión al interior de las instituciones. En suma, aun cuando este instrumento promueve una mejora en la gestión, con especial énfasis en los resultados, se requiere de capacidades que permitan llevar adelante el proyecto.

Desde el punto de vista de las convocatorias de los CDs, es posible que los temas y planes de mejoramiento institucional que se convoquen sean establecidos por las propias instituciones (en una visión estratégica preferentemente corporativa) o por las agencias de financiamiento público, donde se establezcan $e x$ antes objetivos estratégicos específicos nacionales (como reformar el currículo y disminuir la duración de los programas de carrera, o incentivar cambios corporativos para lograr la formación más pertinente y efectiva de profesores) que permitan abordar efectiva y proactivamente ciertas políticas públicas. 


\section{LOS CONVENIOS DE DESEMPEÑO}

Un Convenio de Desempeño es un instrumento de asignación de recursos del Estado a instituciones de educación superior, debidamente seleccionadas. Este instrumento se caracteriza por cuanto la institución que recibe determinados fondos públicos debe asumir el compromiso de alcanzar desempeños académicos notables, negociados con el Ministerio de Educación, y sobre los cuales debe rendir cuenta pública.

El Convenio de Desempeño procura la generación de bienes académicos con alta rentabilidad social. Para la generación de dichos bienes las instituciones de educación superior deben lograr un mejoramiento cualitativo y cuantitativo significativo en algunas de las dimensiones que configuran su quehacer.

Estos convenios se caracterizan por cuanto apuntan a desafíos que se asocian directamente con la calidad institucional y con el cambio estratégico. Están basados en los planes de desarrollo estratégico de las instituciones, e involucran una relación contractual de mediano plazo entre el Ministerio de Educación y la institución beneficiaria. Los montos asignados a las instituciones son significativos y exigen resultados e impactos medibles y verificables de corto y mediano plazo.

Los Convenios de Desempeño como instrumento de asignación de recursos del Estado tienen una serie de rasgos que le distinguen respecto de otras opciones de financiamiento.

En primer lugar, un Convenio de Desempeño financia, esencialmente, bienes públicos, con un compromiso académico expreso de la institución que recibe los recursos, la que debe rendir cuenta pública de los resultados e impactos alcanzados.

En segundo lugar, los Convenios de Desempeño permiten alinear los recursos y capacidades de una institución de educación superior con prioridades nacionales, lo cual permite la generación de sinergias, ya que los recursos asignados se combinan con recursos institucionales y con otras iniciativas estratégicas, generando relaciones causa efecto que permiten una espiral virtuosa de logros.

En tercer lugar, los Convenios de Desempeño no sólo impactan sobre la sociedad mediante la generación de bienes públicos, sino que fortalecen las capacidades institucionales, mejoran la capacidad de gestión de las instituciones, su calidad, así como su eficacia y eficiencia.

En consecuencia, un Convenio de Desempeño debe generar una rentabilidad social positiva, en un marco de transparencia y rendición de cuenta, debida al empleo de los recursos públicos asignados. Además, los Convenios de Desempeño posibilitan el fortalecimiento y el apalancamiento de recursos estratégicos en las instituciones de educación superior, instalando y combinando capacidades cuyo alcance supera el plazo de ejecución del convenio.

Un convenio de desempeño debe generar bienes públicos. En el caso del sistema de educación superior, estos bienes se asocian preferentemente a la formación de capital humano avanzado de pregrado y postgrado; y a la investigación, desarrollo e innovación.

Dado que un convenio de desempeño supone que la institución beneficiaria asumirá compromisos expresos con el Ministerio de Educación y que esos compromisos serán la resultante de un proceso de negociación, cabe preguntarse si este mecanismo de rendición de cuenta resta o no autonomía a las instituciones.

Una primera reflexión al respecto, es que las instituciones tienen la libertad para elegir el área y en las temáticas específicas en las cuales habrán de participar. Su libertad de elección está garantizada. Nadie impone a las instituciones su participación ni la temática que pretenden abordar. Ciertamente, la búsqueda de un acuerdo con el Estado sugiere un proceso voluntario de alineamiento de prioridades estratégicas. La institución no afecta su autonomía por este hecho, probablemente pierde discrecionalidad para emplear los recursos públicos en lo que estime pertinente, pero, por otro lado, la institución gana en transparencia y la sociedad se ve favorecida por la generación de bienes públicos.

Una segunda cuestión es que el gobierno corporativo de la institución participante no es afectado en ninguna medida ni forma. La institución define libremente a los miembros de su comunidad y a los directivos que habrán de participar en el convenio de desempeño. En virtud de lo anterior, 
la autodeterminación está garantizada: el equipo de dirección y el gobierno corporativo no se ven afectados por el convenio de desempeño.

Por lo tanto, las instituciones definen la temática libremente y el equipo que participará en el convenio, con lo cual se conserva, con entera autonomía, su misión institucional y los rasgos que configuran la impronta institucional. Como los recursos que se asignan por Convenios de Desempeño representan solo una parte del financiamiento, otros gastos discrecionales -no elegibles- podrán ser financiados por las instituciones con sus ingresos propios. Este modelo permite entonces a las instituciones disponer de una mezcla de fuentes de financiamiento para llevar adelante planes de mejoramiento institucional.

\section{LOS CONVENIOS DE DESEMPEÑO MECESUP}

Los Convenios de Desempeño piloto del Programa MECESUP (CDs) son contratos que se firman entre el Ministerio de Educación y las instituciones sobre la base de un Plan de Mejoramiento Institucional (PMI) previamente acordado, que establece los objetivos a lograr, las estrategias para llevarlo a cabo y las metas a alcanzar medidas con indicadores de resultados.

La primera convocatoria a las universidades estatales a presentar prepropuestas para el desarrollo de CDs se realizó el año 2006 [7]. Estas propuestas debían considerar la implementación de un PMI, en un plazo de tres años, que comprometiera resultados e impactos mayores que aquellos provistos por los instrumentos convencionales, los que se denominaron desempeños notables o destacados.

Estos primeros CDs se entienden como una experiencia piloto para el desarrollo del instrumento, para su afinamiento y para el aprendizaje del sistema. El objetivo de este instrumento, de acuerdo al Ministerio de Educación, es "alinear las misiones institucionales con las prioridades nacionales y regionales, la autonomía universitaria con la rendición de cuentas públicas (accountability) y el desempeño institucional con el financiamiento público" (Bases Convenios de Desempeño, 2006 [7-8]). Se trata de desarrollar capacidades en las universidades para la gestión orientada a resultados, fortalecer las competencias institucionales en áreas de prioridad nacional, mejorar la calidad de su oferta educacional y comprometer la medición periódica, transparente y pública de sus resultados.

Para orientar a las instituciones se establecieron cinco prioridades ministeriales, a saber, coherencia del PMI con las propuestas que se presentan a concurso; aumento del porcentaje de académicos jornada completa con doctorado; aumento en las tasas de titulación con disminución en el tiempo para lograrlas; incremento en la cobertura de acreditación de programas, y mejoramiento general de la calidad académica.

El PMI debe considerar estrategias que permitan el logro de resultados medibles en el ámbito de la gestión institucional, por lo cual se esperan resultados en las siguientes líneas: instalación y desarrollo de capacidades de análisis institucional, diseño e implementación de sistemas de información para la gestión, aumento de capacidad para el liderazgo institucional, la toma de decisiones basada en evidencia y la planificación estratégica de largo plazo, entre otros. En el segundo eje, calidad académica, se esperan resultados en relación a la mejora de la calidad de los programas, así como su relevancia y pertinencia, incremento de la equidad en el acceso a la calidad, una gestión responsable de la matrícula estudiantil, incremento en la eficiencia, renovación académica, renovación curricular y aumento de la relación universidad-industria.

El CD es un contrato que estipula el objetivo del instrumento y establece, entre otros que:

- Es una asociación entre las partes para el logro de los resultados acordados.

- Se articula en torno al mejoramiento académico y al mejoramiento de las capacidades de gestión.

- Existe un grado de alineamiento explícito entre las prioridades institucionales y las ministeriales.

- Se compromete un mejoramiento notable de la gestión y de la calidad académica.

- Se considera una entrega oportuna de información académica y financiera, así como de los indicadores de desempeño.

- Se apoya en una unidad de análisis institucional para la implementación, monitoreo y seguimiento. 
- Tiene una disposición amplia para la revisión y el monitoreo permanente y sistemático desde el ministerio.

- Genera un anuario de indicadores de desempeño.

Asimismo, el contrato obliga a la institución a cumplir con cada una de las finalidades del PMI y, para cada una de éstas, establecer las líneas de acción, hitos e indicadores de desempeño, los que deberán especificar metas y valores anuales, así como finales. Para la entrega de la segunda cuota y las restantes, se exige la demostración objetiva de hitos y metas en indicadores comprometidos por la universidad en el PMI.

Una característica de los Convenios de Desempeño, que puede resultar clave durante la implementación, es la posibilidad de cambiar dinámicamente la asignación de recursos, buscando maximizar la función objetivo. Esta flexibilidad es fundamental para los propósitos de mejoramiento de desempeño que se persiguen. Como, en general, resulta muy difícil preparar un plan de mejoramiento institucional que sea óptimo para lograr los desempeños notables, resulta clave la retroalimentación y aprendizaje que proporcionan su implementación. Esto ocurre también con la experiencia que pueda provenir de la implementación de otros CDs pertinentes. Los eventuales conflictos que puedan surgir por esta razón al interior de la IES son en general conflictos "buenos"; es decir, los que hay que enfrentar para superar las diferencias (y no quedar "sin acuerdo" como suele ocurrir en muchas otras instancias).

La ejecución del convenio de desempeño se lleva adelante mediante una lista anual de bienes y servicios que permiten ejecutar las tareas que requiere el convenio. En los convenios en ejecución, esta lista ha podido ser modificada, para ajustarse a la realidad del avance del proyecto y a situaciones emergentes.

Para llegar a la versión definitiva del PMI, el ministerio designa un experto o negociador para liderar el proceso de negociación del PMI preliminar. Este proceso se ha develado de gran importancia para mejorar este PMI inicial y definir los desempeños notables que se acuerdan. Los negociadores son claves para acordar metas ambiciosas -pero realistasrespecto de los desempeños (que sean superiores a los que se lograrían con los mismos recursos en una gestión convencional). Los negociadores llevan a las IES a moverse en la frontera. Los negociadores no concluyen su tarea una vez que el contrato se firma, puesto que son los encargados del monitoreo y seguimiento del convenio a lo largo de su implementación y en estas funciones también han sido considerados un importante aporte para el adecuado desarrollo de las iniciativas [1-2].

En la primera convocatoria se seleccionaron prepropuestas de cuatro universidades estatales de diversa índole con el objeto de "verificar el instrumento bajo condiciones lo más diferentes posibles" (Bases Convenios de Desempeño, 2006). Estas universidades son: Universidad de Tarapacá (UTA), Universidad de Chile (UCH), Universidad del Bío-Bío (UBB) y Universidad de La Frontera (UFRO).

Los montos comprometidos en esta iniciativa piloto alcanzan a una inversión pública cercana a los \$10.000 millones. En cuanto a su impacto en el presupuesto de las instituciones, los aportes de los convenios representan ${ }^{9}$ entre un 4,5\% del AFD, en el caso de la Universidad de Chile, y un 30,1\%, en el caso de la Universidad del Bío-Bío. Se puede decir que, en al menos dos casos, UBB y UFRO $(29,1 \%)$, los aportes por concepto de Convenios de Desempeño en relación al AFD son sustantivos. No obstante, si se considera el aporte de los convenios en relación a los ingresos presupuestarios totales de cada universidad, estos fluctúan entre $0,4 \%$ en el caso de la Universidad de Chile y el 2,6\% en el caso de la Universidad de Tarapacá, es decir, son de un impacto presupuestario menor ${ }^{10}$. No obstante esto, de acuerdo a los dos últimos informes del Comité Asesor Internacional del Programa MECESUP y al Informe Internacional de Evaluación de Medio Término, los convenios experimentales se aprecian como un poderoso instrumento para mejorar la gestión institucional.

En cuanto a los objetivos de cada convenio, a pesar de ser, en general, transversales, presentan marcadas especificidades que reflejan las prioridades

9 Fuente: Programa MECESUP2. http://www.mece2.com/ (Convenios de Desempeño)

10 De acuerdo a algunos autores (e.g. García de Fanelli, 2005) los montos involucrados en los convenios deben ser significativos en relación al presupuesto para incentivar los procesos de cambio. 
institucionales. El convenio de la UTA busca posicionar la universidad como agente de integración transfronteriza; el de la UCH instalar un nuevo modelo de gestión institucional; en el caso de la UBB su propuesta se centra en mejorar estructuralmente los niveles de éxito de los estudiantes y, finalmente, el de la UFRO busca un balance entre gestión, eficiencia docente y desarrollo de la investigación en el área de bio-recursos.

Un análisis ${ }^{11}$ de los indicadores de desempeño comprometidos en los convenios y su relación con los indicadores sugeridos en las bases muestra que, a diferencia de los restantes convenios, el de la UCH es uno en el ámbito de la gestión puramente, no comprometiendo resultados académicos. También este análisis refleja que el convenio de la UTA está asociado más a la investigación que al pregrado y, por otro lado, el de la UBB se preocupa del pregrado. En el caso de la UFRO, el convenio involucra resultados en estos dos ámbitos.

El año 2007, la Presidenta de la República anunció recursos especiales para financiar el desarrollo y mejoramiento en las humanidades, artes, ciencias sociales y ciencias de la comunicación en universidades del Estado. Para ello, se decidió su asignación también mediante Convenios de Desempeño.

\section{LECCIONES APRENDIDAS}

Los Convenios de Desempeño presentan desafíos específicos y otros que afectan más generalmente al sector terciario de la educación. Los primeros incluyen características propias del sistema (como su grado de autonomía administrativa y/o de regulación) y del Estado (en materia administrativa). De entre los segundos, se encuentra el grado de estabilidad y coherencia de las políticas y el dominio donde se instalan los instrumentos, es decir, la elegibilidad institucional.

La experiencia desarrollada hasta el momento ha reafirmado que el instrumento provee la oportunidad para un nuevo diálogo entre el gobierno y las universidades. "Estos actores [negociadores] acompañan a las instituciones y al gobierno a lo

${ }^{11}$ Fuente: Bases del Concurso de CD y Convenios de Desempeño de las universidades. http://www.mece2.com/ largo de todo el proceso y ayudan como intérpretes en la alineación de los objetivos entre ambas partes. Ello facilita la convergencia de objetivos entre la agencia (universidades) y el principal (el gobierno)" [3]. Coincidentemente, el informe de marzo de 2009 del Consejo Asesor Internacional (CAI) e Internacional de Medio Término de mayo de 2010 evalúan positivamente el trabajo de los negociadores y la calidad de los procesos de negociación que, según reconocen los directivos de las universidades, les condujo a determinar metas e indicadores de logros exigentes y realistas.

Las inversiones y cambios que se producen en las universidades con la implementación de los convenios involucran nuevos costos recurrentes, los que se buscan morigerar en los procesos de negociación. No obstante este esfuerzo, subsiste la necesidad de mantener nuevas iniciativas instaladas por los proyectos con recursos permanentes. Esto genera presión sobre las autoridades institucionales para hacerse de los ingresos adicionales y dudas sobre la sustentabilidad del instrumento.

El logro de objetivos será uno de los factores más relevantes a la hora de evaluar este instrumento. Esto se operacionalizará midiendo el grado de cumplimiento de los objetivos de cada proyecto institucional. Para lograrlo se recurrirá a los hitos e indicadores, los cuales, hasta el momento, no han sido sometidos aún a una rigurosa prueba.

El seguimiento y monitoreo continuos a lo largo del desarrollo del proyecto (PMI) es una de las fortalezas del diseño chileno, puesto que permite, además, un control permanente de las acciones y del avance de los resultados. Esto contrasta con la experiencia francesa, uno de los países con mayor historia en el desarrollo de este tipo de instrumentos, que evalúa los convenios básicamente cuando se negocia un nuevo contrato [4].

No obstante esta diferencia, la experiencia europea de los contratos programa [4] presenta efectos similares en Chile: se pone de relevancia la asociación entre objetivos y financiamiento, se refuerzan las capacidades institucionales, se consigue especificar y focalizar las propuestas y se coordinan las políticas de evaluación y de financiamiento. 
Un factor que presenta un desafío genérico para la gestión de un convenio de desempeño es "la capacidad de trasladar el mensaje a las unidades operativas de la organización" [3]. En la experiencia chilena, los convenios han sido gestionados y negociados con los equipos de rectoría, por lo que se pueden interpretar como proyectos de la casa central. La estructura de toma de decisiones institucionales y las prioridades de las facultades pueden constituir obstáculos para el alineamiento que requiere la implementación del convenio, por lo que deben preverse estrategias efectivas para involucrar y comprometer el trabajo de las unidades académicas en pos de los objetivos del convenio. Un paso adicional es comprometer, a su vez, el trabajo de los individuos.

Un aspecto que ha afectado la implementación de los Planes de Mejoramiento Institucional incluidos en los CDs, ha sido la dificultad en el manejo de los procesos burocráticos requeridos por el Estado y el Banco Mundial, los que a su vez no siempre han estado bien coordinados entre sí. El retraso en la ejecución afecta alcanzar los hitos comprometidos y dificulta el cumplimiento de las metas anuales. Consciente de esta situación, el informe del CAI [1-2] recomienda que el Estado y las universidades se adapten a las exigencias del nuevo instrumento, aprendan de la experiencia y avancen en la remoción de los obstáculos administrativos.

En relación a esto último, persiste una debilidad estructural a nivel del Ministerio de Educación, que ya ha sido observada en otros contextos, y que recogen los informes internacionales. El amplio y enriquecido diálogo que se produce entre las instituciones y el gobierno, en el marco de un convenio, requieren de personal de alto nivel en el Ministerio de Educación para servir de contraparte a un sector "que se encuentra a la búsqueda de estas nuevas capacidades" [1-2].

Para la buena marcha de un convenio, su dirección debe estar dotada de poder y por lo tanto debe ser parte de la estructura superior de la universidad. Debe exhibir además un liderazgo efectivo. Considerando que quienes detentan cargos superiores tienen poca disponibilidad de tiempo, es necesario profesionalizar el equipo del CD. Además, idealmente radicar los objetivos del CD en el nivel estratégico de la universidad, de modo de alcanzar responsabilidades institucionales.
Por otro lado, se debe lograr una interlocución directa con las unidades operativas, como carreras, direcciones, investigadores, no sólo del equipo técnico y dirección del $\mathrm{CD}$, sino también del negociador y equipo técnico del MECESUP. Llevar un catálogo de acuerdos y avances logrados en las reuniones. Ello genera sentimientos de participación, confianza y evidencias del avance del CD. La comunidad interna de la universidad y los equipos técnicos institucionales deben adquirir conciencia del sentido de los CDs: que no se trata de un proyecto más, sino de un contrato entre el Estado y la universidad que persigue alcanzar objetivos que signifiquen cambios destacados o notables.

En general, el Rector y su equipo directivo han demostrado tener un alto grado de compromiso con el éxito de los CDs. En algunos casos, la calidad del liderazgo del Rector se ha manifestado en niveles superiores, destacándose un estilo transformacional, por demás apropiado a los fines del Convenio de Desempeño.

La conducción "al más alto nivel de la organización", sin necesidad de crear estructuras intermedias ad hoc, ha permitido eliminar barreras burocráticas internas y anclar en el estamento directivo un estilo de gestión centrado en resultados, no como un hecho anómalo, sino que como parte consustancial de administración proactiva.

También se han podido comprometer variaciones amplias y rápidas en áreas de intervención de evolución tradicionalmente lentas y que requieren de intervenciones de largo plazo, en la medida que exista un diseño cuidadoso de los mapas de actuaciones que consideren adecuadamente la cultura organizacional y las cadenas de causalidad que lleven al logro de los indicadores.

Finalmente, las Direcciones de Análisis y Desarrollo Institucional han permitido contar con el soporte analítico para el logro de los resultados comprometidos.

La situación actual de los Convenios de Desempeño en el sistema de educación superior chileno requiere escalar los mismos tanto en términos cuantitativos como cualitativos, para lo cual es fundamental establecer criterios de elegibilidad de instituciones en las cuales es susceptible la implementación de 
este tipo de instrumentos de financiamiento del Estado. Definido el grupo de instituciones que efectivamente está en condiciones de asumir los requerimientos de un convenio de desempeño, es menester configurar en el Ministerio de Educación un equipo de negociadores de alto nivel.

Un primer determinante para el éxito de los Convenios de Desempeño consiste en seleccionar adecuadamente a las instituciones que serán beneficiarias de estos recursos del Estado.

Un segundo elemento a considerar dice relación con el grado de alineamiento entre los propósitos institucionales y los bienes públicos que la institución se ha comprometido a generar por la vía del convenio de desempeño. Los bienes públicos sobre los cuales se acuerda un desempeño notable deben ser esenciales para la institución y en ningún caso marginal para el logro de la misión de la entidad.

Un tercer factor lo constituye el equipo de negociación, el cual debe estar constituido por profesionales de alto nivel, con amplia y exitosa experiencia en alta dirección universitaria, que además cuente con perfiles adecuados para negociar y supervisar entidades complejas y una capacidad de negociar con un profundo conocimiento de las principales cuestiones en juego.

Un cuarto determinante estructural del éxito en la implementación de los Convenios de Desempeño es el liderazgo del Rector de la institución. Se requiere un estilo de liderazgo transformacional y una participación activa y comprometida de las más altas autoridades institucionales.

Un Convenio de Desempeño significa un compromiso de cambio de las estructuras administrativas de ambas partes: la universidad y el Ministerio de Educación de Chile (MINEDUC). En este sentido, es un aprendizaje para ambas, quedando camino por recorrer si se pretende instalar el nuevo instrumento como un mecanismo estable de asignación de recursos. No debe olvidarse, que tres años de implementación es poco tiempo si no se solucionan las limitaciones administrativas que son parte y subsisten en el sistema.

- Sobre la transformación necesaria en el MINEDUC y el Banco Mundial:
La aplicación de los CDs implica no sólo lograr transformaciones de las universidades involucradas, sino que también de las entidades que lo promueven y lideran, en este caso la División de Educación Superior en el MINEDUC y el Banco Mundial.

Los desempeños notables se logran cuando las instituciones y su entorno cambian complementariamente. Si sólo cambian las instituciones, los desempeños no serán lo impactantes que podrían ser.

Por ello, es importante que tanto el MINEDUC, como el Banco Mundial hagan algunas transformaciones en su interior para favorecer un mayor éxito de los CDs. En particular, resulta imprescindible acelerar los ciclos de aprobación de gestión administrativa, facilitar las reprogramaciones, disponer de sistemas de apoyo (por ejemplo, base de datos de especialistas por ámbito) y orientar hacia resultados (y no sólo hacia actividades y procesos).

- Sobre los procedimientos de adquisiciones: Los procedimientos de adquisiciones del Banco Mundial no son del todo apropiados para implementar los CDs, pues retardan innecesariamente la obtención de bienes y servicios. En un CD el tiempo para concretar es clave. Con respecto a Mercado Público, también deben introducirse ajustes para hacerlos apropiados para un CD.

- Sobre el Programa MECESUP y los Negociadores:

Instalar un $\mathrm{CD}$ como una dinámica de búsqueda de resultados notables, lo que orienta a las personas de la universidad a focalizarse en los resultados más que en los procesos y las actividades.

Generar un ambiente de confianza, franqueza y orientación a una transformación genuina. Inducir el trabajo en equipo de los directivos de diversos ámbitos y niveles de la universidad que participan en el CD. El enfoque de "task force" ha producido una colaboración constructiva, sinergia y capacidad para enfrentar dificultades en forma compartida.

Inducir la generación y uso de herramientas para apoyar la gestión del CD (sistema computarizado de seguimiento y control de $\mathrm{CD}$ ). 
- Sobre la universidad beneficiaria:

Los procedimientos administrativos son una variable relevante de gestión para el cumplimiento de los planes de trabajo en la universidad. Para enfrentar las dificultades que suelen generarse, deberían considerarse los siguientes aspectos:

Vincular directamente a quienes ejecutan los actos administrativos en la universidad con el equipo técnico del Programa MECESUP, ojalá a través de reuniones para resolver situaciones concretas.

Adecuar el funcionamiento tradicional de la universidad a las exigencias delCD, particularmente en el cumplimiento de procedimientos y plazos. Reformular los procedimientos internos de la universidad, lo que también sería deseable para el Banco Mundial y el MINEDUC.

Instalar capacidad y disposición para focalizar $\mathrm{y}$ asignar prioridades.

Instalar capacidad y disposición para reasignar recursos según las necesidades de los CDs, en función de los resultados y, en particular, frente a desfases que puedan producirse en la transferencia y recepción de los recursos.

Un desafío que resta por abordar concierne la réplica de sus resultados en otras instituciones. El aprendizaje de los convenios actuales permitirá ahorrar importantes costos económicos y tiempo, y las lecciones aprendidas posibilitarán mayor eficiencia, eficacia y mejores niveles de impacto social. Finalmente, también resulta necesario darle una adecuada continuidad que permita el crecimiento sostenido de los indicadores, evitando que estos se estanquen o decaigan (un escenario que se estima perjudicial para la instalación y escalamiento del instrumento).

\section{CONCLUSIONES}

En base a la experiencia acumulada hasta el momento en el desarrollo de los cuatro Convenios de Desempeño en ejecución, se sugieren aspectos a perfeccionar para que de mejor forma se logre:

- Darle sustentabilidad a este instrumento como política pública.

- Lograr que las universidades adopten una visión y estrategia integradora de su mejoramiento de la calidad en todo su quehacer.
El marco para lograr éxito en la implementación de los Convenios de Desempeño pasa por:

- una adecuada selección de las entidades de educación superior, enfocándose en instituciones con niveles de desarrollo que garanticen una solvencia y capacidad para asumir nuevos desafíos estratégicos;

- la selección de temáticas centrales para el desarrollo de las instituciones y para el cumplimiento de su misión y propósitos;

- un equipo de negociación de alto nivel profesional; $\mathrm{y}$

- un equipo directivo comprometido y motivado con el desafío de lograr resultados notables y de alto impacto.

A continuación se resumen las principales sugerencias y recomendaciones:

- Establecer en las bases del concurso que las instituciones que pueden participar y ser elegible tengan claramente definidos los siguientes aspectos como mínimo:

i) Tener un Plan de Desarrollo Estratégico bien formulado y aprobado oficialmente, junto a un Proyecto Formativo (o Proyecto Educativo), que explicite, entre otros aspectos:

a) El sello que la institución quiere darle a sus egresados.

b) El modelo educativo institucional.

c) Sus políticas a nivel de pregrado, postgrado, investigación y vinculación con el medio, según el tipo de proyecto que formulen.

d) Un sistema de información mínima para la gestión.

ii) Las entidades de educación superior que asuman un convenio de desempeño deben demostrar un nivel inicial satisfactorio en su desarrollo institucional y en su quehacer académico.

iii) Asegurar plenamente el alineamiento del proyecto que presenten las instituciones con las políticas de Educación Superior definidas por el Gobierno.

iv) Tener acreditadas las áreas que presenten en la propuesta de PMI.

v) Tener la organización del equipo del CD alineada con la estructura orgánica de la 
institución, con el fin de que los logros del convenio de desempeño continúen mejorándose una vez terminado el financiamiento externo.

vi) Mejorar los instrumentos de rendición de avance del CD por parte de las instituciones, dándole aún más énfasis a los desempeños notables buscados.

vii) Dar pleno cumplimiento a las fechas de transferencia de los fondos desde el MINEDUC a las instituciones.

viii)Buscar mejoras importantes para agilizar todos los procesos de adquisiciones y llamados a licitaciones, lo cual es particularmente agudo en las instituciones dependientes del Estado, incluyendo al ministerio.

ix) Difundir ampliamente las bases de convocatoria de los Convenios de Desempeño, mediante seminarios u otras instancias, colocando el acento en la diferencia que existe entre este nuevo instrumento y los demás instrumentos de financiamiento, en especial con los proyectos del MECESUP.

x) Difundir ampliamente los Convenios de Desempeño actualmente en ejecución, destacando los aspectos positivos (logros relevantes) y las principales dificultades encontradas.

\section{AGRADECIMIENTOS}

Los autores agradecen el apoyo del Programa Anillo de Investigación en Ciencias Sociales de CONICYT (SOC-01) y del Proyecto FONDECYT 1090116 de CONICYT.

\section{REFERENCIAS}

[1] J.M. Allende, M. Belley, J.J. Brunner, J. Donner, J. González y C. Marquis. "Informes del Comité Asesor Internacional Mecesup2". Mineduc. Febrero 2008 y marzo 2009.

[2] J. Donner, P. Tindemans, F. Marmolejo y J.J. Brunner. "Informe Internacional de Evaluación de Medio Término". Mineduc y BIRF. Mayo 2010.

[3] A.M. García de Fanelli. "Universidad, organización e incentivos. Desafíos de la política de financiamiento frente a la complejidad institucional". Fundación OSDE. Ed. Miño y Dávila. Buenos Aires, Argentina. 2005.

[4] A.M. García de Fanelli. "Los contratosprograma en las universidades: Lecciones de la comparación internacional". Archivos Analíticos de Políticas Educativas. Vol. 14 $\mathrm{N}^{\mathrm{o}}$ 11. Abril 2006. Fecha de consulta: 3 de marzo de 2009. URL: http://epaa.asu.edu/ ojs/article/view/82/208

[5] J. Salmi y M. Hauptman. "Innovations in Tertiary Education Financing: A Comparative Evaluation of Allocation Mechanisms". Banco Mundial, Education Working Paper Series No 4. September, 2006.

[6] J. Salmi y M. Hauptman. "Resource allocation mechanisms in tertiary education". En Higher Education in the World 2006. The Financing of Universities, Houndmills y New York, Palgrave Macmillan. Versión abreviada de la anterior. 2006.

[7] Ministerio de Educación. "Bases administrativas para el concurso de prepropuestas para Convenios de Desempeño". 2006.

[8] Ministerio de Educación. "Prioridades ministeriales y resultados esperados". Convenios de Desempeño. Diciembre 2006. 